\title{
SINTERING OF MLCC'S BARIUM TITANATE WITH MICROWAVES
}

Juan A. Aguilar-Garib, Osvaldo Tijerina-García, Javier Garza-Guajardo

Universidad Autónoma de Nuevo León, FIME, Pedro de Alba s/n, Cd. Universitaria, San Nicolás de los Garza, NL, México

Juan.aguilargb@uanl.edu.mx

Keywords: microwave sintering, barium titanate, MLCC

\begin{abstract}
A comparison of microwave and conventional, in an electric resistance furnace, sintered layers of dielectric base barium titanate $\left(\mathrm{BaTiO}_{3}\right)$ of the kind employed for multilayer ceramic capacitors (MLCC) was performed. Two kinds of samples were used for each processing method; the layers alone without electrodes, and the green MLCC with the layers and electrodes interdigitated. Samples were exposed to microwaves for 20 minutes and heated up to $1050^{\circ} \mathrm{C}$ and $1150^{\circ} \mathrm{C}$ for sintering in a crucible with graphite that acted as reduction agent and microwave susceptor. Conventional sintering was performed in the same arrangement but lasted 120 minutes since it was found that 20 minutes was not enough time to achieve sintering. Heating rate in both cases was $10^{\circ} \mathrm{C} / \mathrm{min}$. It was observed that the layers without the electrodes achieve about the same densification for both processes, while in the case of the green MLCC's the results were variable, ranging from sample that became dust, to cracked samples and some well sintered ones. At least in the microwave case, it is possible that the variability of the results is due to the importance of the location of the sample in the cavity that in turn affects the electric field pattern, especially because the presence of the electrodes that can cause overheating around them.
\end{abstract}

\section{Introduction}

Multilayer ceramic capacitors (MLCC) are one of the most used passive components in consumer electronics. They are fabricated with a base metal electrode technology, where successive layers of dielectric and electrodes. Barium titanate $\left(\mathrm{BaTiO}_{3}\right)$ based ceramic is among the most common dielectric materials employed as dielectric, which is formed in thin layers by type casting of a barbotine. Then the electrodes are printed over the layer, and in turn they are stacked to obtain an interdigitated electrode structure (Figure 1).
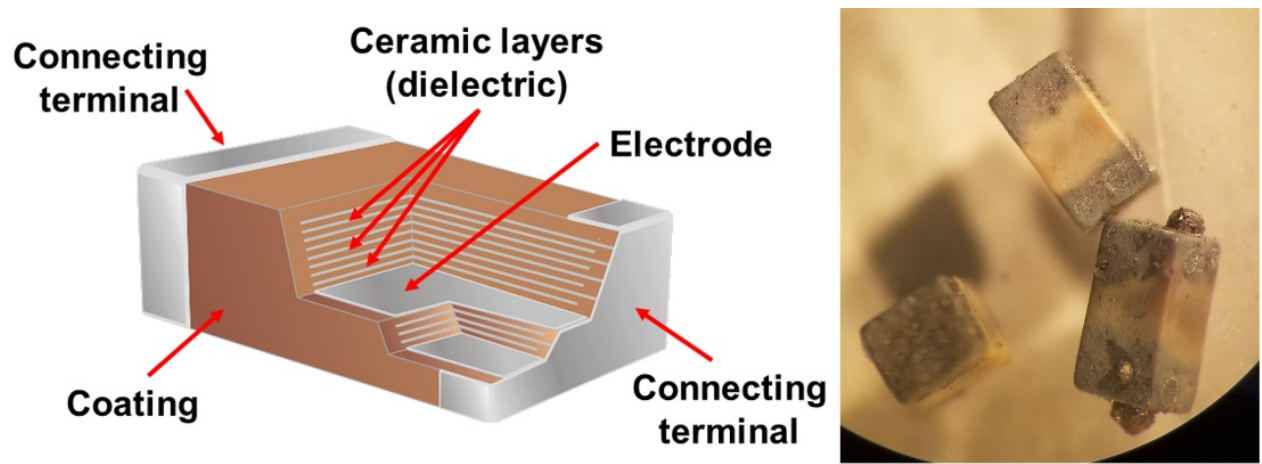

Fig. 1. Scheme of the electrode structure of a MLCC (left). Actual MLCC's, the size is approximately $1.5 \mathrm{~mm}$ X $0.8 \mathrm{~mm}$ X $0.8 \mathrm{~mm}$ (right). 
This structure is then sintered under an atmosphere with the dual purpose of heating and provide reductive conditions to prevent electrode oxidation and the conditions for achieving the final properties of the MLCC.

There are several reports about microwave processing of ceramics [1], some under claims of uniform heat transfer and higher efficiency [2], and reaction rates compared to conventional heating, as well as non-thermal effects of microwaves in the processing [3]. The purpose of this research is to sinter layers of $\mathrm{BaTiO}_{3}$, and the green MLCC's with microwaves and compare them with those sintered conventionally.

One important issue when comparing both processes is that in the conventional case, the maximum temperature and heating rate is governed by conduction of the sample with the boundary conditions dictated by the furnace. The only way for increasing the heating rate is increasing the furnace temperature, but there is the risk to get inappropriate sintering conditions, such as melting of the surface. Heating below this point in conventional heating is relatively easy using the control of the furnace. However, in the case of microwaves, the heating rate is driven by the sample absorption, or the susceptor, hence, often the temperature excesses the set point, and the results obtained from each processing method are different because the thermal conditions where different and it is difficult to raise a conclusion from this kind of tests. Temperature control was considered in the experiments.

Another aspect to consider is that sintering the layers of $\mathrm{BaTiO}_{3}$ with microwaves could resemble the conventional heating, while sintering the MLCC's certainly would produce a different field pattern in the layer due to the metallic electrode, so that the dielectric could exhibit high thermal gradients.

\section{Experimental}

The microwave cavity was feed with a $2.45 \mathrm{GHz}$ magnetron, $1100 \mathrm{~W}$ nominal power, coupled to a WR284 waveguide system with a circulator, dummy load, and a directional coupler, so that forward and reflected powers were registered (Figure 2). The magnetron was taken out from a kitchen oven. These devices use to adjust power output by averaging the on cycling time, keeping the power on for a fraction of time according to the desired power. $50 \%$ nominal power means a $50 \%$ of the time on. In this case the electronics was modified so that that it was possible to adjust the power output all the time that the on/off control dictated according to the heating slope. It is quite common that the actual power of these magnetrons is below the nominal one, and the maximum value is rather variable.

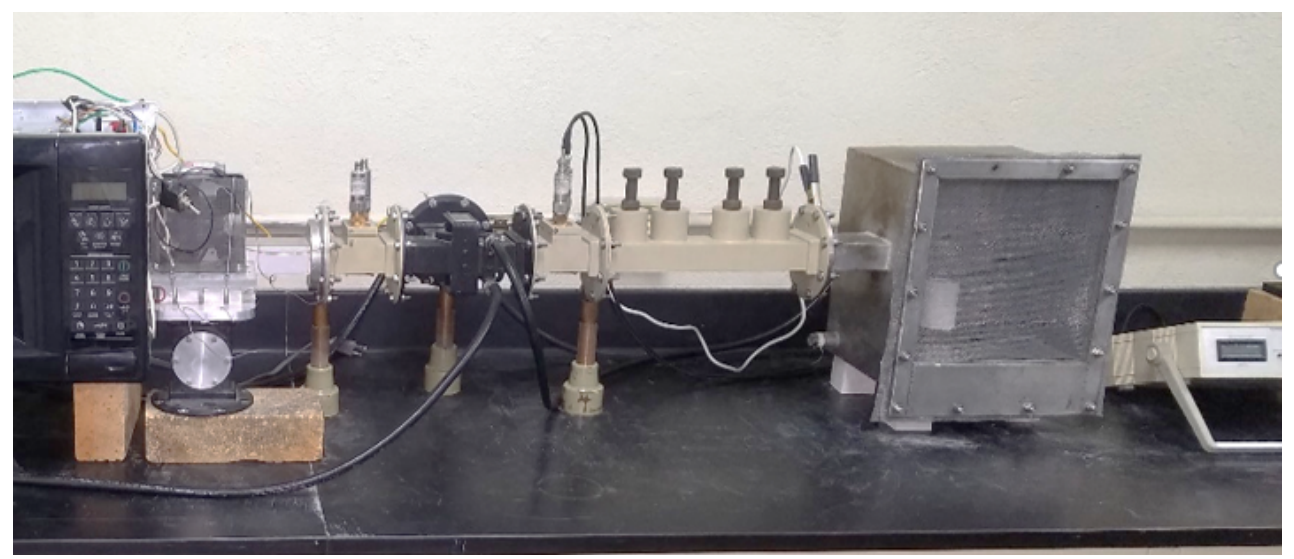

Fig. 2. Experimental arrangement showing the microwave cavity (on the right). 
The temperature was measured with a shield thermocouple and controlled with an on/off device as in reference [1]. It was confirmed that the thermocouple was in such position that did not exhibit self-heating. Optical pyrometry was also employed for having this confirmation. The $\mathrm{BaTiO}_{3}$ was prepared in layers by tape casting and stacked in the same form than the MLCC's, but without the electrodes. The samples of $\mathrm{BaTiO}_{3}$ without electrodes were placed in a crucible with $5 \mathrm{~g}$ of graphite as thermal susceptor and reducing agent, and then set in the microwave cavity for being sintered at $1050^{\circ} \mathrm{C}$ and $1150^{\circ} \mathrm{C}$. These temperatures are often reported in literature [4-6]. The samples were exposed to microwaves for 20 minutes, at a heating rate $10^{\circ} \mathrm{C} / \mathrm{min}$. For conventional heating, the samples were placed in the same crucible with graphite as well in an electric resistance furnace, but in this case, it is only for providing the reduction conditions. It was found that the 20 minutes exposition was not enough for conventional sintering, hence, these tests lasted 120 minutes each with the same heating rate. The green capacitors were treated in the same arrangement and conditions

\section{Results and discussion}

The temperature control was an important issue because the sample was getting hot too quickly so that the proposed heating rate was exceeded. Therefore, it was necessary to adjust the delivered power and then increase it as needed. The appearance of the system heated to $1150^{\circ} \mathrm{C}$ can be seen in figure 3 .

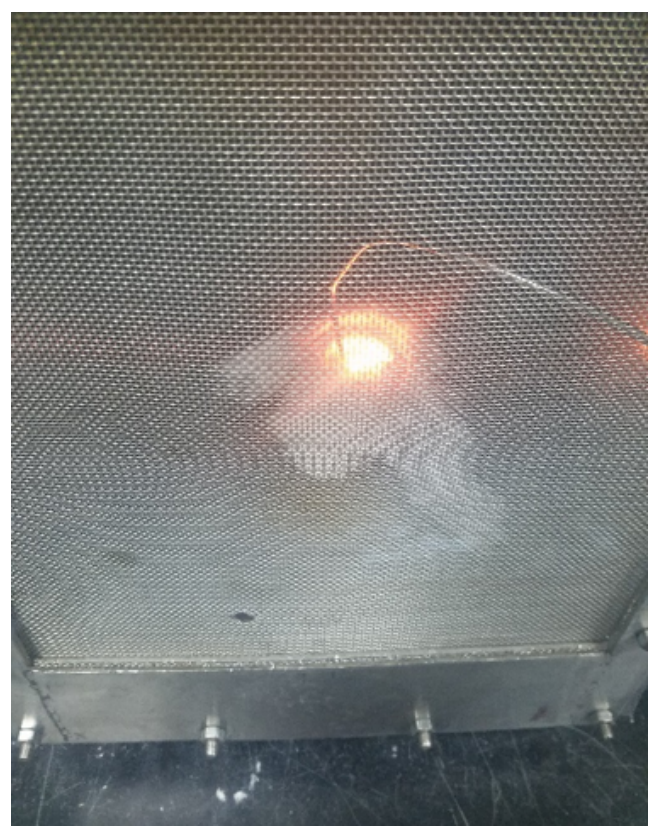

Fig. 3. Appearance of the sample heated at $1150^{\circ} \mathrm{C}$

Figure 4 shows a plot of the earlier stage of heating showing the forward and reflected power against time (sampling time, 3 seconds), while figure 5 shows the whole test but with a sampling time of 1 minute. The cooling time is also here, that is why there is no power at the end, the goal temperature was kept by 20 minutes. 
Notice how forward power is low at the beginning and higher as temperature increased, this adjusted was performed manually. Once that it was possible to control the temperature, the different tests were conducted.

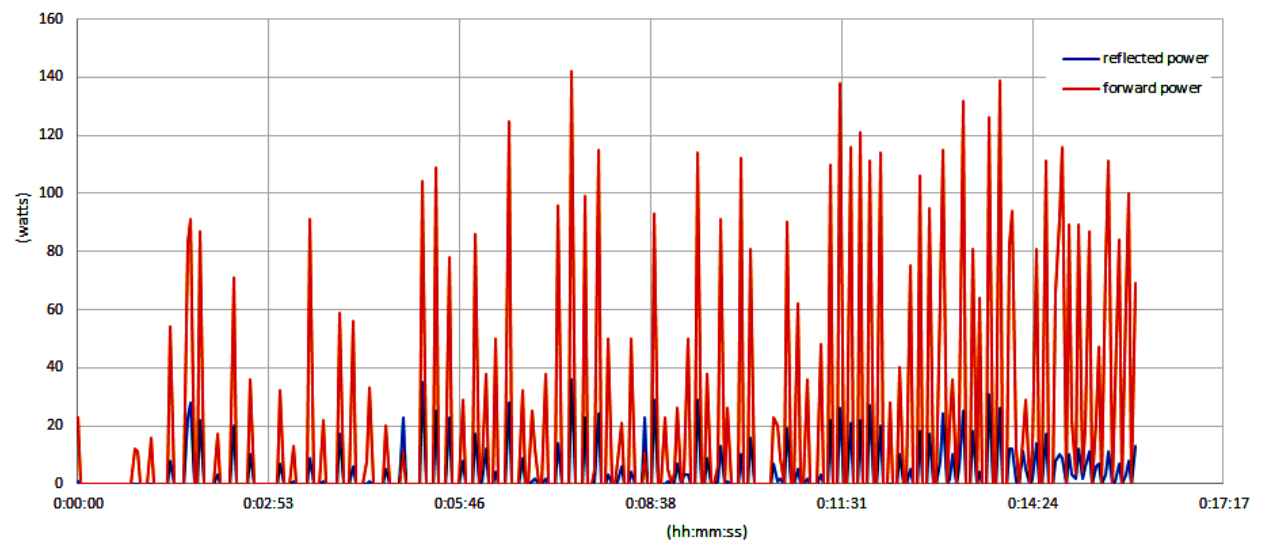

Fig. 4. Example of the measured forward and reflected power during one of the experiments ( 3 seconds of sampling time).

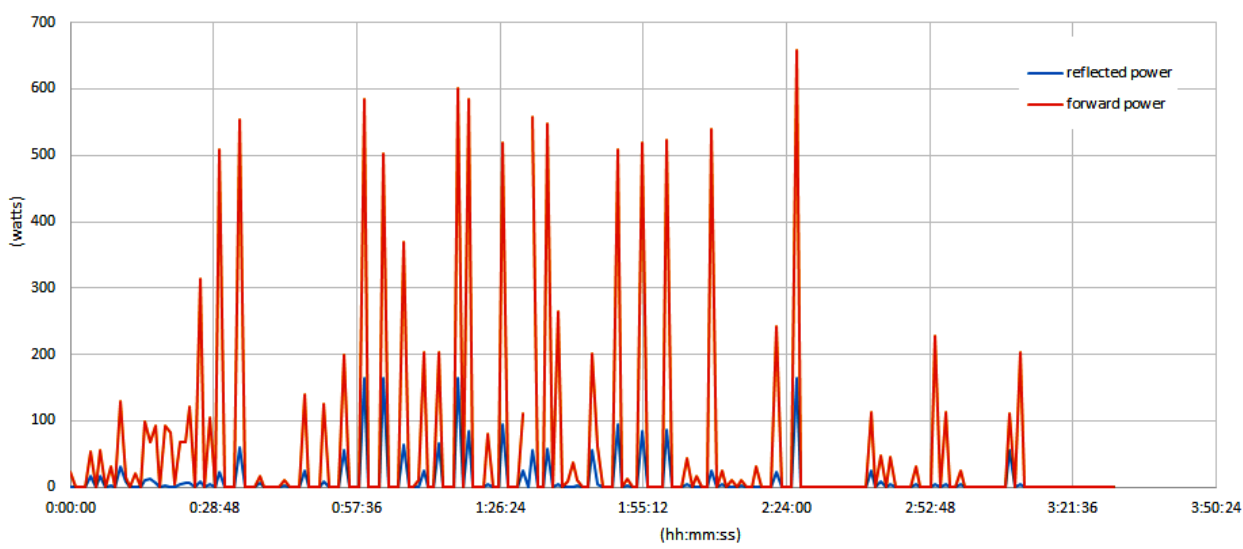

Fig. 5. Example of the measured forward and reflected power during one of the experiments ( 1 minute of sampling time).

The sintering degree can be determined by the rate of achieved density against theoretical density, which in this case is $6.02 \mathrm{~g} / \mathrm{cm}^{3}$. Density was determined by Archimedes method, and the results are shown in table 1.

Table 1. Relative density of the sintered layers.

\begin{tabular}{ccc}
\hline Temperature & Conventional & Microwave \\
\hline $1050^{\circ} \mathrm{C}$ & 0.92 & 0.91 \\
$1150^{\circ} \mathrm{C}$ & 0.88 & 0.92 \\
\hline
\end{tabular}

Sintering the MLCC's produce different results than layers alone, since some of the samples became dust and others developed cracks, just few of them were sintered consistently. Figure 6 shows the aspect of the microwave sample that is sintered with cracks, meaning that this MLCC would not work properly. In this case sintering was compared qualitatively through 
hardness measurements, but they are not conclusive. The same figure shows a MLCC heated in the electric resistance furnace, that also looks sintered, but with a different color. It was expected that only the samples processed in with microwaves would develop cracks, bit as it can be seen in figure 7, the samples processed conventionally also developed them. Since in the microwave case the samples were cooled down at a controlled rate, they were still exposed to microwaves in cycles (figure 5) during cooling. In the conventional case, the samples were cooled down in the furnace. Therefore, it is expected that at least in this late case there are not thermal stresses.
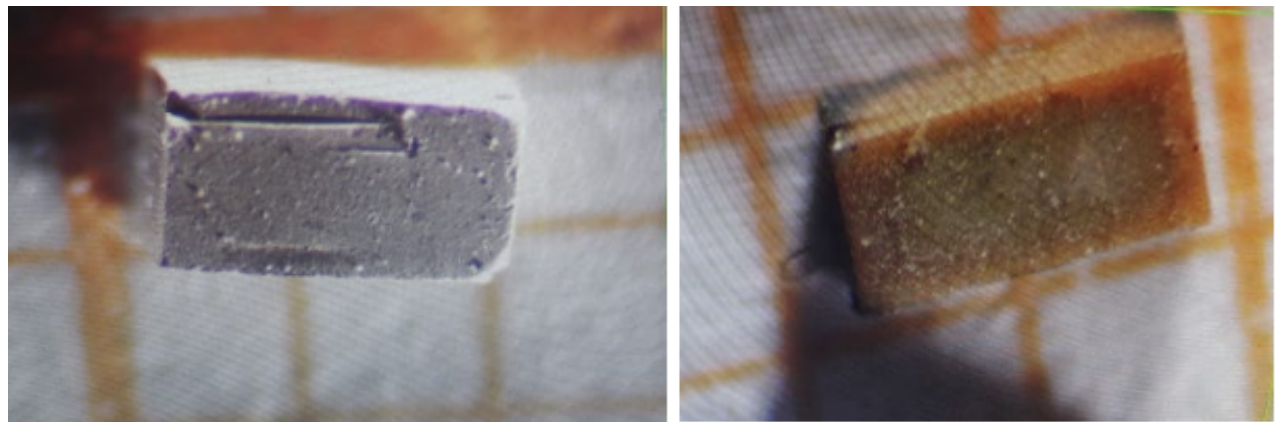

Fig. 6. MLCC sintered with microwaves (left) and conventionally (right). Grid is $1 \mathrm{~mm}$.

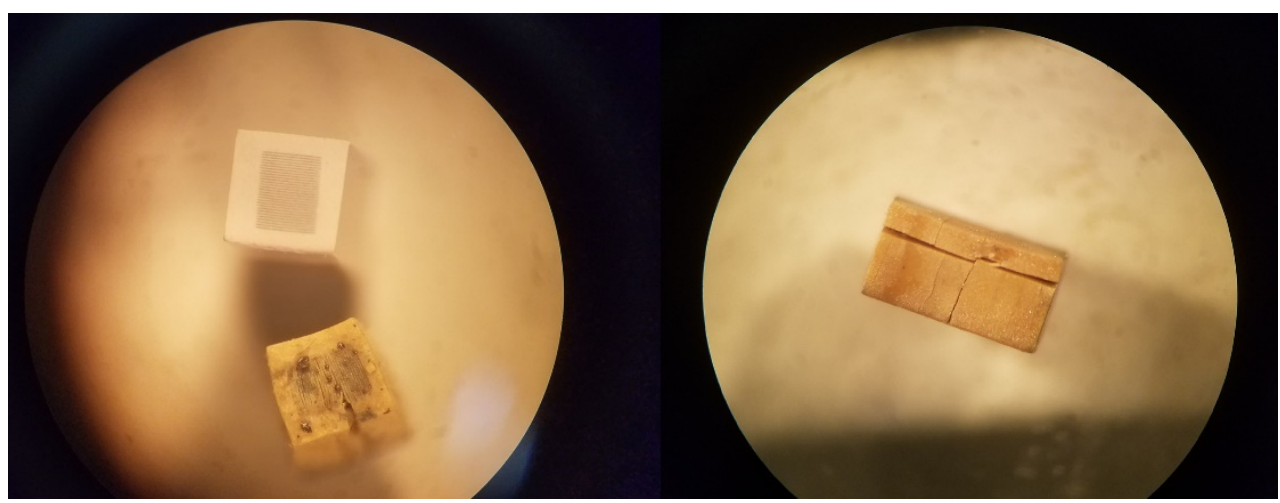

Fig. 7. MLCC sintered with microwaves (left) and conventionally (right).

There are reports of accelerated shrinkage on sintering with microwaves [5]. Despite the combination of having small samples compared to the penetration depth of the microwaves, there is evidence of great thermal gradients in the MLCC's. The temperature that is controlled always is taken in a single location or averaged, then inside the samples the temperatures can be higher. That would explain that sintering takes less time with microwaves than conventionally.

\section{Final remarks}

Although it was confirmed that sintering of ceramics is possible with microwaves, in the case of MLCC's there are several issues that challenges thermal uniformity and the possibility to produce them with microwaves. The only item that was addressed was the achieved density of the ceramic, but still the dielectric properties must be considered for building a capacitor. 


\section{References}

1. J. Aguilar-Garib, Advances in Induction and Microwave Heating of Mineral and Organic Materials, IntechOpen, 2011, 243-268.

2. K. Bai, H-G Kim, Journal of Materials Science Letters, 1994, 13, 806-809.

3. J. Binner, B. Vaidhyanathan, J. Wang, D. Price, M. Reading, Journal of Microwave Power and Electromagnetic Energy, 2008, 42, 47-63.

4. R.K. Patel, P. Kumar, C. Prakash, D.K. Agrawal, Ceramics International, 2012, 38, 1585-1589.

5. S. Derling, H.-P. Abicht, Journal of Microwave Power and Electromagnetic Energy, 1996, 31, 221-227.

6. A. Domer-Reisel, S. Schöps, A. Lenk, G. Schmutzler, Advanced Engineering Materials, 2007, 9, 400-405. 